PROCEEDINGS OF THE

AMERICAN MATHEMATICAL SOCIETY

Volume 125, Number 4, April 1997, Pages 1197-1202

S 0002-9939(97)03627-7

\title{
HOLOMORPHIC HELICES IN A COMPLEX SPACE FORM
}

\author{
SADAHIRO MAEDA AND TOSHIAKI ADACHI \\ (Communicated by Christopher B. Croke)
}

\begin{abstract}
In a complex space form $M$ we shall investigate a smooth curve $\gamma$ which is generated by a holomorphic Killing vector field $X$ on $M$.
\end{abstract}

\section{INTRODUCTION}

In Riemannian Geometry it is interesting to investigate "simple curves" in a certain sense. From this point of view some geometers studied Submanifold Theory (for example, see [1], [2]). In this paper, we consider "simple curves" in a complex space form.

Let $M$ be an $n$-dimensional Kähler manifold with complex structure $J$ and Riemannian metric $\langle$,$\rangle . For a helix \gamma$ on $M$ of order $d(\leq 2 n)$ with the associated Frenet frame $\left\{V_{1}, \ldots, V_{d}\right\}$, we define its complex torsions by $\tau_{i j}(t)=\left\langle V_{i}(t), J V_{j}(t)\right\rangle$ for $1 \leq i<j \leq d$. In the study of helices in a Kähler manifold their complex torsions play an important role. We shall call $\gamma$ a holomorphic helix if all the complex torsions are constant. Ohnita and the first-named author proved in [3] that a smooth curve $\gamma$ on a complex space form is a holomorphic helix if and only if it is generated by a holomorphic Killing vector field $X$. This is the complex version of the well-known fact that a smooth curve on a real space form is a helix if and only if it is generated by a Killing vector field. Study of holomorphic helices is one of the most interesting objects in differential geometry in a complex space form.

The main purpose of this paper is to study the moduli of holomorphic helices of order 3 in complex space forms. A helix of order 1 is nothing but a geodesic, and a helix of order 2 is called a circle. They are necessarily holomorphic helices. But in the class of helices of order $d(\geq 3)$ we can find many helices which are not holomorphic helices.

We show that the moduli of all holomorphic helices of order 3 on an $n$-dimensional complex space form is parametrized by three real numbers or two real numbers according as $n \geq 3$ or $n=2$ (see Theorem 5). Moreover, we investigate the moduli of all holomorphic helices in a 2-dimensional complex space form (see Theorems 4, $5)$.

The authors would like to express their hearty thanks to Professor K. Ogiue for his valuable suggestions during the preparation of this paper. Also, the first-named author is grateful to Professor Y. Ohnita for his kindness.

Received by the editors February 16, 1995 and, in revised form, August 30, 1995

1991 Mathematics Subject Classification. Primary 53C35, 53C20.

Key words and phrases. Holomorphic helix, complex torsion, complex space form.

(C)1997 American Mathematical Society 


\section{Complex torsions of holomorphic Helices}

We shall start by recalling the definition of helices. A smooth curve $\gamma=\gamma(t)$ parametrized by its arc length $t$ is called a helix of proper order $d$ if there exist an orthonormal frame $\left\{V_{1}=\dot{\gamma}, \ldots, V_{d}\right\}$ along $\gamma$ and positive constants $k_{1}, \ldots, k_{d-1}$ which satisfy the system of ordinary differential equations

$$
\nabla_{t} V_{j}(t)=-k_{j-1} V_{j-1}(t)+k_{j} V_{j+1}(t), \quad j=1, \ldots, d,
$$

where $V_{0}=V_{d+1}=0$ and $\nabla_{t}$ denotes the covariant differentiation along $\gamma$. The constants $k_{j}(1 \leq j \leq d-1)$ and the orthonormal frame $\left\{V_{1}, \ldots, V_{d}\right\}$ are called the curvatures and the Frenet frame of $\gamma$, respectively. A curve is called a helix of order $d$ if it is a helix of proper order $r(\leq d)$. For a helix of order $d$ which is of proper order $r(\leq d)$, we use the convention in (1.1) that $k_{j}=0(r \leq j \leq d-1)$ and $V_{j}=0(r+1 \leq j \leq d)$. Note that every helix is a real analytic curve on a Kähler manifold $M$.

As a matter of course complex torsions of helices satisfy $\left|\tau_{i j}(t)\right| \leq 1$ by their definitions. We first show that complex torsions and curvatures of holomorphic helices have relations. Differentiating all the complex torsions, we find by use of the equation (1.1) that

$$
\frac{d}{d t} \tau_{i j}(t)=-k_{i-1} \tau_{i-1, j}(t)+k_{i} \tau_{i+1, j}(t)-k_{j-1} \tau_{i, j-1}(t)+k_{j} \tau_{i, j+1}(t),
$$

where $\tau_{k l}=0$ when $k=l$ or $k=0$ or $l$ is greater than the proper order. We hence get the following.

Proposition 1. The complex torsions of a holomorphic helix of odd proper order $d$ on a Kähler manifold satisfy the following relations:

$$
\begin{aligned}
\tau_{i, i+2 k} & =0 \text { for } i=1,2, \ldots, d-2 k, \text { where } k=1,2, \ldots,(d-1) / 2, \\
k_{1} \tau_{2 d} & =k_{d-1} \tau_{1, d-1}, \\
k_{1} \tau_{2 j}+k_{j} \tau_{1, j+1} & =k_{j-1} \tau_{1, j-1} \text { for } j=3,5, \ldots, d-2, \\
k_{i-1} \tau_{i-1, d}+k_{d-1} \tau_{i, d-1} & =k_{i} \tau_{i+1, d} \text { for } i=3,5, \ldots, d-2, \\
k_{i-1} \tau_{i-1, j}+k_{j-1} \tau_{i, j-1} & =k_{i} \tau_{i+1, j}+k_{j} \tau_{i, j+1} \\
& \quad \text { for } i=2,3, \ldots, d-3, j=i+2, i+4, \ldots, d-1 .
\end{aligned}
$$

Proposition 2. The complex torsions of a holomorphic helix of even proper order $d$ on a Kähler manifold satisfy the following relations:

$$
\begin{aligned}
\tau_{i, i+2 k} & =0 \text { for } i=1,2, \ldots, d-2 k, \text { where } k=1,2, \ldots,(d-2) / 2, \\
k_{1} \tau_{2 d} & =k_{d-1} \tau_{1, d-1} \\
k_{1} \tau_{2 j}+k_{j} \tau_{1, j+1}= & k_{j-1} \tau_{1, j-1} \text { for } j=3,5, \ldots, d-1, \\
k_{i-1} \tau_{i-1, d}+k_{d-1} \tau_{i, d-1}= & k_{i} \tau_{i+1, d} \text { for } i=2,4, \ldots, d-2, \\
k_{i-1} \tau_{i-1, j}+k_{j-1} \tau_{i, j-1}= & k_{i} \tau_{i+1, j}+k_{j} \tau_{i, j+1} \\
& \quad \text { for } i=2,3, \ldots, d-3, j=i+2, i+4, \ldots, d-1 .
\end{aligned}
$$

Conversely, if the Frenet frame of a helix $\gamma$ in a Kähler manifold satisfies the above relations at a point, then all $n$th derivatives of its complex torsions vanish at this point. Since $\gamma$ is real analytic, we find that it is a holomorphic helix. We therefore have 
Proposition 3. For orthornormal vectors $v_{1}, \ldots, v_{d}$ at a point $p$ of a Kähler manifold $M$, we set $\tau_{i j}=\left\langle v_{i}, J v_{j}\right\rangle(1 \leq i<j \leq d)$. If positive constants $k_{1}, \ldots, k_{d-1}$ and the vectors $v_{1}, \ldots, v_{d}$ satisfy the relations in Proposition 1 or 2 , there exists a unique holomorphic helix with curvatures $k_{1}, \ldots, k_{d-1}$ satisfying that the initial value of its Frenet frame is $\left(v_{1}, \ldots, v_{d}\right)$.

The following is easily verified.

Proposition 4. The complex torsions $\tau_{i j}$ of a holomorphic helix of proper order d on a Kähler manifold $M$ satisfy $\sum_{j=1}^{i-1} \tau_{j i}^{2}+\sum_{j=i+1}^{d} \tau_{i j}^{2} \leq 1$ for every $i$.

Proof. Since $\tau_{i, i+2 k}=0$, we find that $\left\{V_{2 l-1}, J V_{2 l-1} \mid l=1,2, \ldots,\right\}$ and $\left\{V_{2 l}, J V_{2 l} \mid\right.$ $l=1,2, \ldots\}$ form orthornormal frames. When $i$ is even, the left-hand-side of the inequality is the norm of the projection of $V_{i}(0)$ onto the complex linear subspace spanned by $\left\{V_{2 l-1}(0), J V_{2 l-1}(0) \mid l=1,2, \ldots\right\}$. We hence get the inequality. For odd $i$ we have a similar argument.

Here we treat holomorphic helices of order 3 . We need to choose orthonormal vectors $v_{1}, v_{2}, v_{3} \in T_{p} M$ which satisfy

$$
k_{1}\left\langle v_{2}, J v_{3}\right\rangle=k_{2}\left\langle v_{1}, J v_{2}\right\rangle,\left\langle v_{1}, J v_{3}\right\rangle=0 .
$$

Identifying $T_{p} M$ with $C^{n}$, we set $v_{1}, v_{2}$ and $v_{3}$ as

$$
\begin{aligned}
& v_{1}=(1,0, \ldots, 0), \\
& v_{2}=\left(-i \tau, \sqrt{1-\tau^{2}}, 0, \ldots, 0\right), \\
& v_{3}=\left(0,-i \rho / \sqrt{1-\tau^{2}}, \sqrt{1-\tau^{2}-\rho^{2}} / \sqrt{1-\tau^{2}}, 0, \ldots, 0\right)
\end{aligned}
$$

for positive constants $\tau$ and $\rho$ with $\tau^{2}+\rho^{2} \leq 1$. Then they are orthonormal and satisfy $\left\langle v_{1}, J v_{2}\right\rangle=\tau,\left\langle v_{2}, J v_{3}\right\rangle=\rho,\left\langle v_{1}, J v_{3}\right\rangle=0$. We therefore have

Theorem 1. Let $M$ be a Kähler manifold of dimension greater than 2. Then the following hold:

(1) Every holomorphic helix of order 3 satisfies

$$
k_{1} \tau_{23}=k_{2} \tau_{12}, \tau_{13}=0,\left|\tau_{12}\right| \leq k_{1} / \sqrt{k_{1}^{2}+k_{2}^{2}} .
$$

(2) Conversely, if nonnegative constants $k_{1}, k_{2}$ and a constant $\tau$ satisfy $|\tau| \leq$ $k_{1} / \sqrt{k_{1}^{2}+k_{2}^{2}}$, then there exists a holomorphic helix of order 3 on $M$ with the first curvature $k_{1}$ and the second curvature $k_{2}$, and with the first complex torsion $\tau_{12}=\tau$. $M$.

(3) If $|\tau|>k_{1} / \sqrt{k_{1}^{2}+k_{2}^{2}}$, we have no such a holomorphic helix of order 3 on

Theorem 2. Let $M$ be a 2-dimensional Kähler manifold. Then the following hold:

(1) The complex torsions of each holomorphic helix of proper order 3 in $M$ are

$$
\tau_{12}=k_{1} / \sqrt{k_{1}^{2}+k_{2}^{2}}, \tau_{13}=0, \tau_{23}=k_{2} / \sqrt{k_{1}^{2}+k_{2}^{2}}
$$

or

$$
\tau_{12}=-k_{1} / \sqrt{k_{1}^{2}+k_{2}^{2}}, \tau_{13}=0, \tau_{23}=-k_{2} / \sqrt{k_{1}^{2}+k_{2}^{2}},
$$

where its curvatures are $k_{1}$ and $k_{2}$.

(2) Conversely for given positive constants $k_{1}$ and $k_{2}$, there exists a holomorphic helix of proper order 3 with curvatures $k_{1}$ and $k_{2}$, and with complex torsions defined by (1.2) or (1.3). 
Such a description as above for holomorphic helices of order 4 is much more complicated. We restrict ourselves here to holomorphic helices on a 2-dimensional Kähler manifold $M$. For given constants $\tau$ and $\rho$ with $\tau^{2}+\rho^{2}=1$, we choose vectors

$$
v_{1}=(1,0), v_{2}=(-i \tau, \rho), v_{3}=(0,-i), v_{4}=\mp(i \rho, \tau)
$$

in $T_{p} M \simeq C^{2}$. Then they are orthonormal and satisfy

$$
\begin{gathered}
\left\langle v_{1}, J v_{2}\right\rangle=\tau,\left\langle v_{2}, J v_{3}\right\rangle=\rho,\left\langle v_{1}, J v_{4}\right\rangle= \pm \rho \\
\left\langle v_{1}, J v_{3}\right\rangle=\left\langle v_{2}, J v_{4}\right\rangle=0,\left\langle v_{3}, J v_{4}\right\rangle= \pm \tau
\end{gathered}
$$

On the other hand, Proposition 2 shows that a helix is a holomorphic helix if and only if

$$
\begin{aligned}
\tau_{13}(0)= & \tau_{24}(0)=0, k_{1} \tau_{23}(0)+k_{3} \tau_{14}=k_{2} \tau_{12}(0), \\
& k_{1} \tau_{14}(0)+k_{3} \tau_{23}(0)=k_{2} \tau_{34}(0) .
\end{aligned}
$$

We therefore have

Theorem 3. Let $M$ be a 2-dimensional Kähler manifold. Then the following hold:

(1) The complex torsions of each holomorphic helix of proper order 4 with curvatures $k_{1}, k_{2}$ and $k_{3}$ on $M$ satisfy one of the following:

$$
\tau_{12}=\tau_{34}=\tau, \tau_{23}=\tau_{14}=k_{2} \tau /\left(k_{1}+k_{3}\right), \tau_{13}=\tau_{24}=0,
$$

where $\tau= \pm\left(k_{1}+k_{3}\right) / \sqrt{k_{2}^{2}+\left(k_{1}+k_{3}\right)^{2}}$,

$$
\tau_{12}=-\tau_{34}=\tau, \tau_{23}=-\tau_{14}=k_{2} \tau /\left(k_{1}-k_{3}\right), \tau_{13}=\tau_{24}=0,
$$

when $k_{1} \neq k_{3}$, where $\tau= \pm\left(k_{1}-k_{3}\right) / \sqrt{k_{2}^{2}+\left(k_{1}-k_{3}\right)^{2}}$, or

$$
\tau_{12}=\tau_{34}=\tau_{13}=\tau_{24}=0, \tau_{23}=-\tau_{14}= \pm 1
$$

when $k_{1}=k_{3}$.

(2) Conversely, for given positive constants $k_{1}, k_{2}$ and $k_{3}$, there exist holomorphic helices of proper order 4 in $M$ with curvatures $k_{1}, k_{2}$ and $k_{3}$, and with complex torsions defined by (1.4), (1.5) or (1.4), (1.5').

\section{Moduli of holomorphic Helices on A COMPlEX SPACE FORM}

Let $M_{n}(c)$ be an $n$-dimensional complete simply connected complex space form of constant holomorphic sectional curvature $c$. It is well-known that an arbitrary complex space form is locally complex analytically isometric to a complex projective space, a complex hyperbolic space or a complex Euclidean space according as the holomorphic sectional curvature $c$ is positive, negative or zero. We have the following holomorphic congruence theorem for helices in $M_{n}(c)$.

Proposition 5 ([3]). Let $\gamma$ and $\sigma$ be two helices of orders $p$ and $q$ in a complex space form $M_{n}(c)$, respectively. Let $\left\{k_{1}, \ldots, k_{p-1}\right\}$ (resp. $\left.\left\{\lambda_{1}, \ldots, \lambda_{q-1}\right\}\right)$ be the curvatures of $\gamma($ resp. $\sigma)$, and let $\tau_{i j}^{\gamma}(t)$ (resp. $\left.\tau_{k l}^{\sigma}(t)\right)$ be the complex torsions of $\gamma$ (resp. $\sigma$ ). Then there exists a holomorphic isometry $\varphi$ of $M_{n}(c)$ satisfying $\gamma=\varphi \circ \sigma$ if and only if $p=q, k_{i}=\lambda_{i}(1 \leq i \leq p-1)$ and $\tau_{i j}^{\gamma}(0)=\tau_{i j}^{\sigma}(0)(1 \leq i<j \leq p)$.

In this section we denote by $H h^{d}\left(M_{n}(c)\right)$ the set of the equivalence classes of all holomorphic helices of order $d(\leq 2 n)$ in $M_{n}(c)$ with respect to holomorphic isometries of $M_{n}(c)$. By Proposition 5 the set $H h^{d}\left(M_{n}(c)\right)$ is naturally regarded as a subset of $[0, \infty)^{d-1} \times[-1,1]^{d(d-1) / 2} \subset R^{(d+2)(d-1) / 2}$. Needless to say, every 
helix which lies on a totally real totally geodesic submanifold $M^{n}(c / 4)$ of $M_{n}(c)$ is a holomorphic helix (whose complex torsions are zero in $M_{n}(c)$ ), so that the set of the equivalence classes of all holomorphic helices of proper order $d(\leq n)$ with respect to holomorphic isometries of $M_{n}(c)$, which is a subset of $H h^{d}\left(M_{n}(c)\right)$, is not empty.

Theorem 4. For given positive constants $k_{1}, k_{2}$ and $k_{3}$, there exist four equivalence classes of holomorphic helices of proper order 4 with curvatures $k_{1}, k_{2}$ and $k_{3}$ with respect to holomorphic isometries of $M_{2}(c)$. In addition, these four equivalence classes are given by (1.4), (1.5) or (1.4), (1.5').

We give some examples here of holomorphic helices of proper order 4 in a 2-dimensional complex projective space $C P_{2}(c)$. Let $\pi: S^{2 n+1}(1)\left(\subset C^{n+1}\right) \rightarrow$ $C P^{n}(4)$ denote the Hopf fibration.

Example 1. For any $k$ satisfying $0<k<\sqrt{2}$, we put

$$
\begin{aligned}
& A=\sqrt{\left(4-k^{2}-\sqrt{\left(2-k^{2}\right)\left(8-k^{2}\right)}\right) /\left(2\left(8-k^{2}\right)\right)}, \\
& B=2 / \sqrt{8-k^{2}}, \\
& C=\sqrt{\left(4-k^{2}+\sqrt{\left(2-k^{2}\right)\left(8-k^{2}\right)}\right) /\left(2\left(8-k^{2}\right)\right)} \\
& \alpha=\left(\sqrt{2-k^{2}}+\sqrt{8-k^{2}}\right) / \sqrt{2}, \\
& \beta=\sqrt{2-k^{2}} / \sqrt{2} \\
& \delta=\left(\sqrt{2-k^{2}}-\sqrt{8-k^{2}}\right) / \sqrt{2} .
\end{aligned}
$$

Let $\tilde{\gamma}$ be a curve in $C^{3}$ defined by $\tilde{\gamma}(t)=\left(A e^{i \alpha t}, B e^{i \beta t}, C e^{i \delta t}\right)$. Then $\tilde{\gamma}$ is a horizontal curve on $S^{5}(1)$ parametrized by arc length $t$. Moreover $\pi(\tilde{\gamma})$ is a holomorphic helix of order 4 in $C P_{2}(4)$ with curvatures $k_{1}=k, k_{2}=\sqrt{\left(18-9 k^{2}\right) / 2}$ and $k_{3}=k$, and with complex torsions $\tau_{12}=\tau_{13}=\tau_{24}=\tau_{34}=0, \tau_{14}=1$ and $\tau_{23}=-1$. The curve $\pi(\tilde{\gamma})$ satisfies $\left(1.5^{\prime}\right)$.

Example 2. Let $\tilde{\gamma}$ be a curve in $C^{3}$ defined by

$$
\tilde{\gamma}(t)=\left((1 / \sqrt{3}) e^{i t},(1 / \sqrt{14}) e^{2 i t},(5 / \sqrt{42}) e^{-4 i t / 5}\right) .
$$

Then $\pi(\tilde{\gamma})$ is a holomorphic helix of order 4 in $C P_{2}(4)$ with curvatures $k_{1}=3 \sqrt{2} / 5$, $k_{2}=11 \sqrt{2} / 10$ and $k_{3}=1 / \sqrt{2}$, and with complex torsions $\tau_{12}=\tau_{14}=\tau_{23}=\tau_{34}=$ $-1 / \sqrt{2}$ and $\tau_{13}=\tau_{24}=0$. The curve $\pi(\tilde{\gamma})$ satisfies (1.4).

Finally we shall investigate the moduli spaces $H h^{d}\left(M_{n}(c)\right)(d=1,2,3)$. The moduli space $H h^{1}\left(M_{n}(c)\right)$ clearly consists of one point. As an immediate consequence of Theorem 1, Theorem 2 and Proposition 5 we can establish the following.

Theorem 5. (1) The moduli space $H h^{2}\left(M_{n}(c)\right)$ is homeomorphic to a cone in $R^{2}$ or a half line according as $n \geq 2$ or $n=1$. More precisely, $H h^{2}\left(M_{n}(c)\right)$ is $[0, \infty) \times[-1,1] / \sim$ or $[0, \infty)$ according as $n \geq 2$ or $n=1$, where the equivalence relation $\sim$ means that $(0, \tau) \sim(0, \rho)$ if $\tau, \rho \in[-1,1]$. 
(2) The moduli space $H h^{3}\left(M_{n}(c)\right)$ is connected and

$$
\begin{aligned}
& H h^{3}\left(M_{n}(c)\right) \\
& =\left\{\begin{array}{l}
\left\{\left(k_{1}, k_{2}, \tau\right) \in[0, \infty) \times[0, \infty) \times[-1,1] \mid \tau^{2} \leq k_{1}^{2} /\left(k_{1}^{2}+k_{2}^{2}\right)\right\} / \sim, \quad n \geq 3, \\
([0, \infty) \times\{0\} \times[-1,1] \\
\left.\quad \cup\left\{\left(k_{1}, k_{2}, \pm k_{1} / \sqrt{k_{1}^{2}+k_{2}^{2}}\right) \mid k_{1}>0, k_{2}>0\right\}\right) / \sim, \quad n=2,
\end{array}\right.
\end{aligned}
$$

where the equivalence relation $\sim$ means that $(0, k, \tau) \sim(0, l, \rho)$ if $k, l \in[0, \infty)$ and $\tau, \rho \in[-1,1]$.

Remark. Let $\gamma$ be a holomorphic helix of proper order 3 with curvatures $k_{1}$ and $k_{2}$, and with the first complex torsion $\tau_{12}=\tau$ in a complex space form $M_{n}(c)$. Then $\gamma$ lies on a totally real totally geodesic submanifold $M^{3}(c / 4)$ of $M_{n}(c)$ if and only if $\tau=0$, and $\gamma$ lies on a holomorphic totally geodesic submanifold $M_{2}(c)$ of $M_{n}(c)$ if and only if $\tau= \pm k_{1} / \sqrt{k_{1}^{2}+k_{2}^{2}}$.

\section{REFERENCES}

1. D. Ferus and S. Schirrmacher, Submanifolds in Euclidean space with simple geodesics, Math. Ann. 260 (1982), 57-62. MR 83j:53002

2. S. L. Hong, Isometric immersions of manifolds with planar geodesics into Euclidean space, J. Diff. Geom. 8 (1973), 259-278. MR 52:9122

3. S. Maeda and Y. Ohnita, Helical geodesic immersions into complex space forms, Geometriae Dedicata 30 (1989), 93-114. MR 90b:53067

Department of Mathematics, Shimane University, Matsue, Shimane, 690, Japan

E-mail address: smaeda@botan.shimane-u.ac.jp

Department of Mathematics, Nagoya Institute of Technology, Gokiso, Nagoya, 466, JAPAN

E-mail address: d43019a@nucc.cc.nagoya-u.ac.jp 